Authors

John L. Hall, Jun Ye, Scott A. Diddams, Long-Sheng Ma, Steven T. Cundiff, and David J. Jones 


\title{
Ultrasensitive Spectroscopy, the Ultrastable Lasers, the Ultrafast Lasers, and the Seriously Nonlinear Fiber: A New Alliance for Physics and Metrology
}

\author{
John L. Hall, Jun Ye, Scott A. Diddams, Long-Sheng Ma, Steven T. Cundiff, and David J. Jones
}

Invited Paper

\begin{abstract}
We now appreciate the fruit of decades of development in the independent fields of ultrasensitive spectroscopy, ultrastable lasers, ultrafast lasers, and nonlinear optics. But a new feature of the past two or three years is the explosion of interconnectedness between these fields, opening remarkable and unexpected progress in each, due to advances in the other fields. For brevity, we here focus mainly on the new possibilities in the field of optical frequency measurement.
\end{abstract}

Index Terms-Femtosecond lasers, frequency control, frequency synthesizers, measurement, optical frequency comb, optical frequency measurement, stabilized laser.

\section{INTRODUCTION AND OVERVIEW}

$\mathbf{S}$ YSTEMATIC work in standards labs worldwide, over thirty years, has shown reliable progress in the art of stable lasers and measurement of their frequencies. By now, lasers are controlled in the $\delta f / f \sim 10^{-15}$ domain using numerous attractive quantum resonators, including $\mathrm{Yb}^{+}, \mathrm{In}^{+}$, $\mathrm{Sr}^{+}, \mathrm{Hg}^{+}$, and $\mathrm{Mg}, \mathrm{Ca}, \mathrm{Sr}, \mathrm{I}_{2}, \mathrm{CH}_{4}, \mathrm{OsO}_{4}, \mathrm{C}_{2} \mathrm{HD}$, and $\mathrm{C}_{2} \mathrm{H}_{2}$. In the past, a few major labs have developed articulated chains of phase-related oscillators, organized to phase-coherently connect optical oscillators locked to these narrow optical resonances with frequency standards in the microwave/RF domain. In the meantime, an independent research community has evolved reliable self-mode-locked lasers for ultrafast science, based on the remarkable properties of the Ti:Sapphire laser. Still another group has developed special photonic-crystal optical fibers for tight confinement and dispersion-free optical propagation over a wide wavelength band. The completely unexpected development of the last two years is the confluence

Manuscript received April 20, 2001; revised May 14, 2001. This work was supported in part by the National Institute of Standards and Technology, the National Aeronautics and Space Administration, and the National Science Foundation.

J. L. Hall, J. Ye, and S. T. Cundiff are with JILA, Boulder, CO 80309-0440 USA (e-mail: jhall@jila.colorado.edu).

S. A. Diddams was with JILA, Boulder, CO 80309-0440 USA. He is now with the Time and Frequency Division, National Institute of Standards and Technology, Boulder, CO 80303 USA.

L.-S. Ma was with JILA, Boulder, CO 80309-0440 USA. He is now with the Department of Physics, East China Normal University, Shanghai, China.

D. J. Jones was with JILA, Boulder, CO 80309-0440 USA. He is now with PhotonEx, Bedford, MA 01730 USA.

Publisher Item Identifier S 0018-9197(01)10048-5. of these independent currents into a new and beautiful reality in which all these technical advances combine to make a frequency comb of octave bandwidth with some 3 million frequency comb components of well-known frequencies. For measuring optical frequencies, this single-laser self-calibrating frequency synthesizer is ideal, both for metrologists interested in frequency standards and as well for the physicist interested in the properties of some special quantum transition in a special element. The ultrafast community, in return, has received a bounty from the merger in that it can now use the new frequency-domain-based tools to control femtosecond pulse generation with accurately controlled carrier-envelope relative phase. Recent work also shows the capability of frequency methods to stabilize the timing of femtosecond laser pulses; even pulses of different repetition rates emitted by separate lasers. Precision frequency-offset phase locking between separate ultrafast lasers has also become possible. The near future will show explosive growth of these synergies in scientific applications, and in the stimulation of practical designs including, perhaps, use of more nonlinear base materials for photonic crystal fibers. Surely this will continue to be an exciting time.

\section{INTRODUCTION TO THE SPECTROSCOPY}

\section{A. Background and Scientific Interest}

This paper is intended mainly to be a summary of the most remarkable recent progress in the field of stabilized lasers and their measurement. But it may be useful to quickly sketch the history of this field which began in the mid 1960s with the stabilization of a laser to its Doppler gain center. Soon, the narrower Lamb dip in a pure neon discharge was preferred for its narrower linewidth. Then, almost immediately, separation of the optical-gain and optical-frequency reference functions led to nice molecular absorber resonances a thousand-fold narrower than their Doppler linewidths. A then-contemporary view of this epoch is available [1]. Over the next 30 years, numerous experimental tricks have been introduced to improve $S / N$, resolution, accuracy of modulation, utilization of high-frequency modulation, etc., leading to improved performance as optical frequency standards. Many molecular absorbers have been investigated, driven by accessibility with convenient or well-performing lasers, and by physical issues such as preferring large 
mass to reduce transit broadening and relativistic Doppler shifts. As tunable lasers developed, one could begin to think of atomic systems which would be especially suitable or meaningful to investigate, mainly $\mathrm{H}, \mathrm{D}, \mathrm{He}$, positronium, and muonium. Optical cooling techniques soon appeared, followed shortly by optical trapping, and have led to the preparation of atomic samples with approximately a million atoms at such small velocities $(\sim<1 \mathrm{~m} / \mathrm{s})$ that milliKelvin or microKelvin may be the appropriate temperature scales. The currently active list of favorite atomic systems includes basically everything about $\mathrm{H}$ [2], $\mathrm{He}$ [3], and weak/narrow "clock" transitions in $\mathrm{Hg}^{+}$[4], [5], $\mathrm{In}^{+}$[6] $\mathrm{Sr}^{+}$[7], $\mathrm{Yb}^{+}$[8], Bi [9], Ag [10], Ca [11], [12], $\mathrm{Mg}$ [13], Sr [14], [15], and Ba. Resonance natural widths $\delta \nu$ in the few kilohertz to the sub-hertz domain are, in principle, available according to one's selection of a favorite atomic transition, corresponding to a natural decay time $\tau$ in the $100-\mu \mathrm{s}$ to 1-s domain. In principle, one could obtain about $1 /(2 \tau)$ interactions per second with approximately only twofold broadening by the interrogation process. So, if we collect all the available information-bearing photons, for a single measurement a $S / N \sim \sqrt{ } N$ should be available. Normalizing to a standard 1 -s measurement time gives us $S / N \sim \sqrt{ } N * \sqrt{ }(1 / 2 \tau)$. An optimum frequency control system could find the center of the resonance with a precision $\sim 1 /(S / N)$ in $1 \mathrm{~s}$. Taking the resonance linewidth into account then leads to a frequency uncertainty $\delta f(1 s) \sim \delta \nu /(S / N)=(2 / N \tau)^{1 / 2}$. Just as an example, the $400-\mu$ s lifetime of the $\mathrm{Ca}^{1} \mathrm{~S}_{0}{ }^{-3} \mathrm{P}_{1}$ transition at $657 \mathrm{~nm}$ ideally could provide a $<100 \mathrm{mHz}$ laser stability at $1 \mathrm{~s}$, using $10^{6}$ atoms. Noting that the transition frequency is $456 \mathrm{THz}$, this leads to a rather attractive $1.6 \times 10^{-16}$ projected fractional stability! A stability within a factor of 25 of this ideal value has recently been reported [12]. No wonder people have become interested in this art form. Of course, so many atoms in a small volume may bring some problems, collisional shifts, etc. Another approach is to use just a single ion, such as $\mathrm{Hg}^{+}$with $\tau \sim 160 \mathrm{~ms}$, which leads to a possible stability of about $1.8 \mathrm{~Hz}$ at $1 \mathrm{~s}$ [4]. The transition frequency in this case is $1065 \mathrm{THz}(\lambda \sim 282 \mathrm{~nm})$, so one can expect $\sim 2 \times 10^{-15}$ stability at $1 \mathrm{~s}$ [5]. Different issues affect the accuracy of various stabilized laser systems based on atom traps or on single trapped ions, so many different systems are under active study worldwide. For example, with a single trapped $\mathrm{Yb}^{+}$ion, the electric octupole ${ }^{2} \mathrm{~S}_{1 / 2}{ }^{-2} \mathrm{~F}_{7 / 2}$ transition has been estimated to have a lifetime of $\sim 10$ years [16]. So, at least line $Q$ will not be the main problem in this case.

\section{B. Measurements of Simple Atoms}

Turning to focus upon resonances in simple atomic systems, of course we first must consider hydrogen. As a frequency standard, atomic hydrogen is probably not an ideal choice: it is basically too hard to make and keep as an atom, too sensitive to electric fields, too light and too fast-moving at usual temperatures. But it is simply the fundamental atom and so it is essential-let us add fascinating - to study. Prof. Häensch's development of hydrogen atom spectroscopy has been a spectacular story for more than a quarter century, down to the present linewidth in the range of $1 \mathrm{kHz}$, basically limited by the gas temperature of a few degrees kelvin [17]. The recent achievement of BEC in hydrogen by Prof. Kleppner's group at MIT provides a won- derfully low temperature, although the density may be too high for the best spectroscopic precision [18]. But this is the sign of a problem that we are glad to welcome to the lab. At present, it seems reasonable to expect line-center accuracy for the hydrogen $1 \mathrm{~S}-2 \mathrm{~S}$ transition in the dozens of hertz range, out of the (two photon transition) frequency of $2500 \mathrm{THz}$, corresponding to an (in-) accuracy in the range of a few parts in $10^{14}$ [17]. Unfortunately, even before this level, unwelcome complexity such as the extended character of the proton's charge distribution intervene [19].

Helium [3] offers an access to the study of splittings associated with the spin-orbit interaction, which are interesting in view of their dependence upon the fine-structure constant $\alpha \cong$ 1/137. A representative total experiment would measure Rydberg series over both singlet $2^{1} \mathrm{~S}$ and triplet $2^{3} \mathrm{~S}$ metastables states which would lead to values for their energies and separations.

\section{Prototype Optical Frequency Standards}

Turning to optical frequency standards per se, one type of promising approach can be based on overtone transitions of simple molecules [20]. For H-containing organic molecules, the "designer" features include a choice of color range: $3.2 \mu \mathrm{m}$, $1.5 \mu \mathrm{m}, 1030 \mathrm{~nm}, 790 \mathrm{~nm}, 640 \mathrm{~nm}$, i.e., vibrational changes by $1,2,3,4,5$ quanta. Overtones $(\Delta v>1)$ have shorter transition wavelengths, but suffer corresponding consequences of weakening the transition strength by a factor $\sim 100-10000$-fold. A good feature is that the natural lifetime is some few milliseconds for all of these fundamental and overtone transitions, limited by $\Delta v=-1$ fluorescence near $3 \mu \mathrm{m}$. Thus, narrow lines could be possible, except that the thermally moving molecules just fly right on through our light beam, and thus terminate their coherent interaction. One direct approach is to magnify the beam diameter, giving a $1-\mathrm{kHz}$ linewidth for $w_{0}=8 \mathrm{~cm}$ using a $\mathrm{CH}_{4}$ transition, which was sufficient to resolve the recoil splitting [21]. Also, if one has adequate $S / N$, a beautiful tradeoff is possible by using lower laser power to narrow these lines; this occurs because only the slowest molecules can experience much saturation [22]. Velocity selection to narrow the line by 13-fold was demonstrated in $\mathrm{C}_{2} \mathrm{H}_{2}$ at JILA [23], and optical widths in the 10- $\mathrm{Hz}$ domain for the $\mathrm{CH}_{4} 3.39-\mu \mathrm{m}$ fundamental line were achieved by Bagayev using both slow molecule selection and expanded light beams [24]. New understanding and a new modulation strategy has led to dramatically improved detection sensitivity for weak transitions in molecules located within a high finesse cavity [23], [25]. This so-called "NICE-OHMS" method has produced a record sensitivity of $5 \times 10^{-13}$ integrated absorption, in a 1-s averaging time. Applied to the extra-weak combination band $\nu_{2}+3 \nu_{3}$ in $\mathrm{C}_{2} \mathrm{HD}$, it was possible to obtain a measurement $S / N$ of 7670 in a $1 \mathrm{~s}$ averaging time, leading to a measured laser frequency (in-)stability of $2 \times 10^{-13}$ at $1 \mathrm{~s}$. A 70-fold stronger $\mathrm{C}_{2} \mathrm{H}_{2}$ transition is at $1037 \mathrm{~nm}$ in the $3 \nu_{3}$ band, leading us to expect an even better $S / N$ when laser source issues are addressed. A first observation of, and locking onto this resonance, was recently reported [26].

Our "final" stabilized laser prototype for this discussion is the Nd: YAG monolithic laser, frequency-doubled and locked onto transitions in molecular $\mathrm{I}_{2}$ near $532 \mathrm{~nm}$. For example, using 


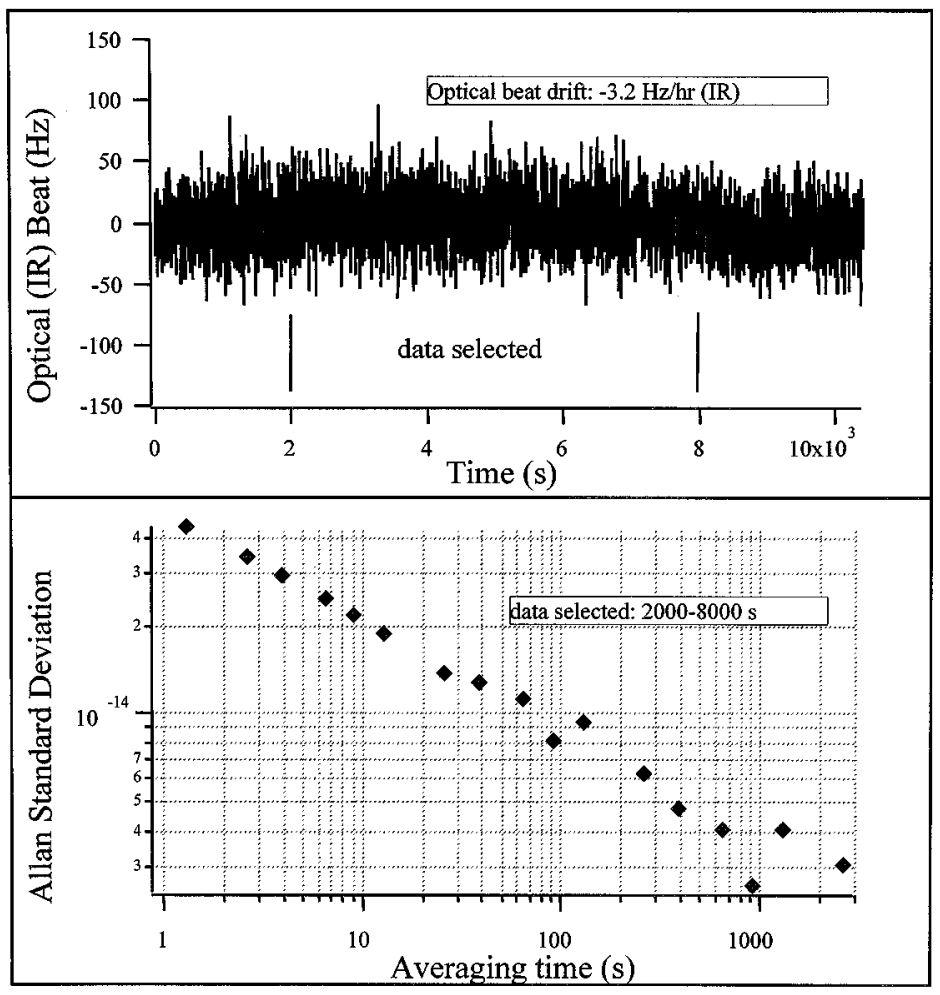

Fig. 1. Beat record between two comparable $\mathrm{I}_{2}$-stabilized Nd lasers. Servo control of the modulator's RAM was switched on at $t=0$. Other laser's modulator was not stabilized.

modulation transfer spectroscopy with the $a_{10}$ hyperfine structure component of the $\mathrm{R}(56) 32-0$ band of ${ }^{127} \mathrm{I}_{2}$, we obtain a $S / N$ of 8700 in $1 \mathrm{~s}$, corresponding to an (in-)stability of $4 \times$ $10^{-14}$ for each $\mathrm{I}_{2}$-stabilized laser $(\sim 25 \mathrm{~Hz})$ [27]. Fig. 1 shows the measured time record of the beat frequency between two comparable systems [28]. The average drift over the record is $3.2 \mathrm{~Hz} / \mathrm{h}$ in the IR. This is not what we will want for a fundamental standard yet, but it is not a bad intermediate result. The beginnings of a high-resolution iodine frequency atlas were indicated [27]. Study of these several hyperfine spectra revealed an interesting rotational variation of the electric quadrupole coupling constant [29].

Of course, other groups are busy as well and Fig. 2 shows the present stability versus averaging time for a number of the best optical and RF sources. Of particular note are the $\mathrm{OsO}_{4}$ results where $\sim 1 \times 10^{-13}$ stability over some years has been shown by absolute frequency measurements [30]. The first phase-coherent frequency measurement into the visible domain $(657 \mathrm{~nm})$ was the absolute frequency of $\mathrm{Ca}$ atoms stored in a magnetooptic trap, reported [11] by the Physikalisch-Technische Bundesanstalt (PTB), Braunschweig, Germany, colleagues in 1996. Then, the National Research Council (NRC) chain was extended in Ottawa, Canada [7] in 1999 to reach the $\mathrm{Sr}^{+}$transition at 674 $\mathrm{nm}$. Also in 1999, the $\mathrm{In}^{+}$transition at $236.5 \mathrm{~nm}$ was measured at the Max Planck Institute for Quantum Optics [6]. An initial frequency measurement has been reported for $\mathrm{Yb}^{+}[8]$. The recent frequency measurement of $\mathrm{H} 1 \mathrm{~S}-2 \mathrm{~S}$ in Garching was based on comparison with the transportable Cs fountain clock built by the Laboratoire primaire du Tems et des Frequences (LPTF) and the Ecole normale Superieure, Paris, France. The hydrogen measurement represents an unprecedented (in-) accuracy of only $1.8 \times 10^{-14}$ [17]. Recent excellent results on $\mathrm{Hg}^{+}$ and $\mathrm{Ca}$ at NIST [31] push this frontier even further, to $1.0 \times$ $10^{-14}$ for $\mathrm{Hg}^{+}$.

\section{OPTICAL-MicROWAVE CONNECTION}

\section{A. Traditional Optical Frequency Chains}

In brief, the second point of this paper is that, despite the excellent frequency stability obtainable in the optical domain, it has had but little impact in the frequency standards community. That was because - until the last year of the last millennium-there was basically no realistic way to accurately connect the optical and microwave domains. Of course, there have been a few harmonic synthesis frequency chains built up in national metrology labs. But the scale of required effort has been the same for 28 years: approximately five people and five years for a single precise optical measurement. A review of all such frequency chain measurements was given by Jennings [32] and recently updated by Knight [33]. Recent measurements of this type include the $\mathrm{Ca}$ transition by PTB [11] and the $\mathrm{Sr}^{+}$frequency by the NRC (Ottawa, Canada) [7]. In Garching, H [34] and $\mathrm{In}^{+}$frequency measurements were made with a closely related optical frequency interval divider chain [35].

\section{B. Direct Synthesis-The Femtosecond Way}

These measurements were hard, detailed work. Now, after the breakthrough of using femtosecond laser frequency combs, frequency measurement is "easy." The new millennium and femtosecond techniques have changed all our previous step-by-step 


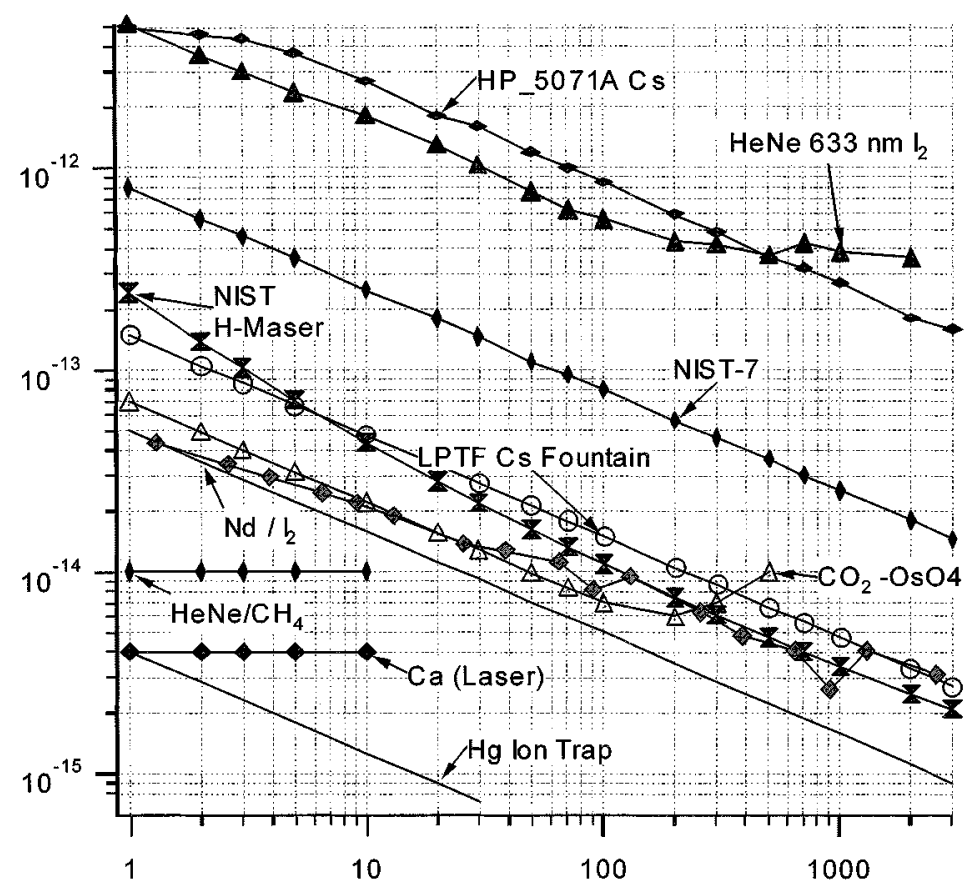

Fig. 2. Allan frequency stability of current best frequency sources. Some curves without points indicate expected performance levels, based on their short-term measured stability. Unfortunately, new noise sources can contribute at longer times, as may be seen in the $\mathrm{CO}_{2}-\mathrm{OsO}_{4}$ and $\mathrm{Nd} / \mathrm{I}_{2}$ experimental curves. The $\mathrm{Ca}$ (laser) curve shows basically only the instability of the laser's reference cavity.

approaches. Now we can make a "rough" frequency measurement of an unknown stable CW optical source in just minutes. "Rough" in this context means accuracy better than one part in a million million: $<1 \times 10^{-12}$. This incredible progress results from independent progress in diode-pumped intensity-stable solid-state pump laser technology, from development of the remarkable $\mathrm{Ti}$ : sapphire laser with self-organizing mode locking [36], explanation of its origin as a time-dependent Kerr-lens gating [37], refinement of its design [38] to produce adequately short and powerful pulses and, in the latest stage, the availability of special small-core microstructured optical fiber [39] with designer dispersion characteristics-most notably zero group velocity dispersion near the 800-nm laser pulse centroid. The laser pulses are energetic enough that, with their $\sim 10$-fs duration, peak powers exceed a quarter MW. The fiber core is small enough that this power maps into an intensity of a quarter $\mathrm{TW} / \mathrm{cm}^{2}$, which produces a serious level of nonlinearity. We estimate the peak nonlinear phaseshift is more than 30 radians during passage through $15 \mathrm{~cm}$ of microstructure fiber. The pulse envelope goes from zero to maximum and back in about $12 \mathrm{fs}$, corresponding to a "modulation" bandwidth in the $100-\mathrm{THz}$ range. So, this deep, fast, phase-modulation process is destined to blast FM sidebands all across the visible and near IR. Following Ranka et al. [40], we can observe "white" light being produced by the magic rainbow fiber. The reason that this drastic violence is interesting and useful in metrology is that it is strictly repetitive, thanks to the stable repetition rate of the Kerr-lens mode-locked laser and the high-amplitude stability of its output. Clearly, with such a deep nonlinear modulation, to suppress AM noise, we need each successive femtosecond peak to be the same intensity to within a tolerance of $1: 10^{3}$. If there were more amplitude variation between pulses, we would not have each pulse exiting with its spectral phases stable to $\ll 1$ radian. This suggests the remarkably fragile and delicate parameter space we have to enjoy.

The useful connection between the repetitive pulse train and its frequency implications appears to have been realized at almost the same time by Hänsch in Garching and Chebotayev in Novosibirsk. Basically, the rigorous periodicity in time will mean a periodicity in the spectral domain: the spectrally broadband "white" light is not white at all, but rather is intensely structured, if only we use adequate resolution to see the structure. Because the pulse's time domain shape contains exceedingly little power in between the pulses, the Fourier spectrum shows hardly any power in between the repetitive spectral peaks, which we can call the "femtosecond Laser Comb." Prof. Hänsch made use of a "picosecond laser" spectral comb for frequency measurements already in a 1978 publication [41]. The current epoch began in 1999, when "The Garching" group used the natural comb produced by a commercial 70-fs laser, with its spectral span of $\sim 20 \mathrm{THz}$. This corresponds to $\sim 10 \%$ fractional bandwidth. Their first femtosecond comb experiment used the $\mathrm{CH}_{4}$-stabilized laser as the absolute frequency reference for a measurement of the $\mathrm{Cs}_{1}$ line [42]. By twice doubling the $\mathrm{CH}_{4}$ frequency, one comes to $848 \mathrm{~nm}$, where heterodyne measurement established the location of the whole comb relative to methane. Then at $895 \mathrm{~nm}$ another heterodyne with comb lines established the location of the $\mathrm{Cs}_{1}$ line's laser relative to the comb, albeit at some 244000 comb lines' distance. Using a filter cavity to select one mode in every 20 soon made clear the exact integer number of the comb lines involved and thus the unknown frequency.

The next step was to use the Frequency Interval Divider concept [35] for a new measurement of the H 1 S-2 S transition frequency. It happens that the needed optical frequency at 486 $\mathrm{nm}$ is near seven times the $\mathrm{CH}_{4}$ standard, so the Garching chain 
already had a laser at 1/2 the 486-nm frequency, i.e., at $972 \mathrm{~nm}$, which is about $44 \mathrm{THz}$ from the 848-nm reference. Basically, the scheme [43] was to use two different synthesis factors $(4 \times$ and $3.5 \times$ ) with the known $\mathrm{CH}_{4}$ IR standard to define known visible frequency locations, and then measure their separation in terms of comb-line intervals, i.e., the measurable repetition frequency. In effect, one was measuring the $\mathrm{CH}_{4}$ standard as well as the hydrogen transition. Thus, here for the first time, one had a direct synthesis from RF to the optical domain, since the frequency of the 3.39- $\mu \mathrm{m}$ reference laser could itself be determined by counting comb lines. But the approach did need phase-coherent loops and auxiliary lasers to regenerate the two optical harmonics of the reference laser, and also some interval divider stages because the comb from the laser was marginally "narrow"-a fractional bandwidth of "only" $10 \%$ or so.

\section{Controlling the Frequencies of the Femtosecond Comb}

We have argued that a periodic temporal behavior is mapped into a spectrum with equally spaced components, separated by the temporal pulse-repetition frequency $f_{\text {rep }}$. This is true. However, the comb lines are not necessarily harmonics of the repetition rate or, equivalently, a huge extension of the comb toward zero frequency would reveal that there is a frequency offset $f_{\text {ceo }}$ of the closest comb lines from zero frequency. How can this happen? This situation is a consequence of our being a little too quick in stating that the pulses are exactly periodic. Actually, the laser cavity contains air and a laser crystal with an index of refraction much different from zero. So we are sure the cavity phase- and group-velocities are unequal. But this is not so important, since the cavity length of $\sim 1 \mathrm{~m}$ supports many modes. What counts is the group velocity dispersion, which operates to return different spectral components around the cavity with differing time delays. Considering the enormous bandwidth of the pulse, there must be some dispersion of the materials comprising the laser system, and it is necessary to provide compensation, via optical tricks [44]. A prism pair, with judicious choice of spacing, can cancel the large linear index term (phase quadratic with frequency) by careful adjustment of the insertion depth of one of the compensating prisms. Thoughtfully matching and an optimum choice of the Ti : sapphire crystal length with the chosen prism material can result in effective reduction of the next (cubic) phase term, as shown by Murnane and Kapteyn [38]. These techniques taken together result in stable Kerr-lens mode-locked operation of the laser, with a minimum pulsewidth $\sim 10$ fs.

So, in summary, the narrow pulses are obtained when the group velocity dispersion is minimized across the pulse's frequency spectrum. But this criterion does not strongly control the other dimension for laser oscillation: the oscillation of each frequency ordinarily corresponds to a Phase-Velocity eigenmode. Since, in general, we have phase velocity $\neq$ group velocity, we can see that the envelope function will not be stable with respect to the underlying optical oscillation frequencies. The result is that there is a phase slip between "carrier" phase and the envelope peak for each of the successive pulses emitted by the laser. Denoting this pulse-to-pulse slip phase by $\varphi$, we will have each optical comb frequency to be given by $f_{N}=N f_{\text {rep }}+f_{\text {ceo }}$. This corresponds to the slipping rate $f_{\text {ceo }}=f_{\text {rep }} * \varphi / 2 \pi$. Here, $N$ represents the integer $\left(\sim 5 \times 10^{6}\right)$ harmonic number of the optical comb line relative to the repetition rate. Also, it is worth noting that we estimate the total carrier-envelope phase shift to be some ten's of cycles: $\varphi$ represents the noninteger remainder part of this phase.

An important technology challenge is to be able to servo-control the repetition rate $f_{\text {rep }}$ and the frequency offset $f_{\text {ceo }}$ independently. Dual to the view of $f_{\text {rep }}$ as the pulse repetition rate is the view of $f_{\text {rep }}$ as the (common) beat frequency between the modes. As shown in Fig. 3, an elegant scheme was applied by Prof. Hänsch's group to control this frequency: to make the beat frequencies larger between adjacent cavity modes, basically one wants to introduce a phase-shift that increases proportional to the frequency displacement of the considered mode [43]. Such a result is obtained naturally by delicately rotating the flat mirror in the spectrally dispersed intracavity region located after the prism pair [45]. An adequate differential distance per mode is introduced well before the mirror is macroscopically rotated out of optical alignment, and yields a tuning response $\delta f_{\text {rep }}=k \beta$. If the pivot of the twist were in the midpoint of the dispersed spectrum, the angle change $\theta$ would produce a change of the repetition rate nearly independent of the "carrier," i.e., central optical frequency $f_{c}$. If the pivot point is displaced, some length change will also be coupled into the change in $f_{c}$, and so also into $f_{\mathrm{CeO}}$.

The other degree of freedom can initially be taken to be the cavity length, which will typically be tightly locked using a reasonably fast piezo transducer. This "piston" action will produce a global extension of each of the standing wave patterns by the same distance $\delta l$, with resulting frequency shifts $\delta f / f=-\delta l / l$, whereas the "twister" action changes $f_{\text {rep }}$, and so every comb line shifts by an equal amount relative to its neighbor. Thus, the "twister"-induced shift of all comb components will be scaled by their respective distance from the pivot point. The optimum method of orthogonalizing these corrections is still a subject of investigation. For an example, see [57].

\section{OpticAl BANDWidTh BEyOnd 1 OCTAVE}

\section{A. The Femtosecond Laser Alone}

Our Ti : sapphire laser provided pulses of $\sim 12$ fs and a bandwidth $\sim 180 \mathrm{~nm}$, centered near $800 \mathrm{~nm}$. Yet our superstable $\mathrm{Nd}$ : YAG laser had its fundamental output at $1064 \mathrm{~nm}$, just out of range. Following the fiber-based broadening results of others, we were pleased to find the output pulse bandwidth extended usefully to the red after passing a few meters of standard 800-nm fiber. This was sufficient to enable beats to be obtained between the femtosecond laser comb and the 1064-nm reference laser, across a 104-THz interval [46]. Then, the known Rb reference at $778 \mathrm{~nm}$ could serve as frequency standard to determine the $\mathrm{Nd}$ frequency. To make sure of the spatial collimation, both fields were propagated within the same fiber which led us to become aware that the strong impulse could write sidebands onto any $\mathrm{CW}$ beam it was traveling with. Additional measurements showed the origin to be cross-phase modulation [47]. 


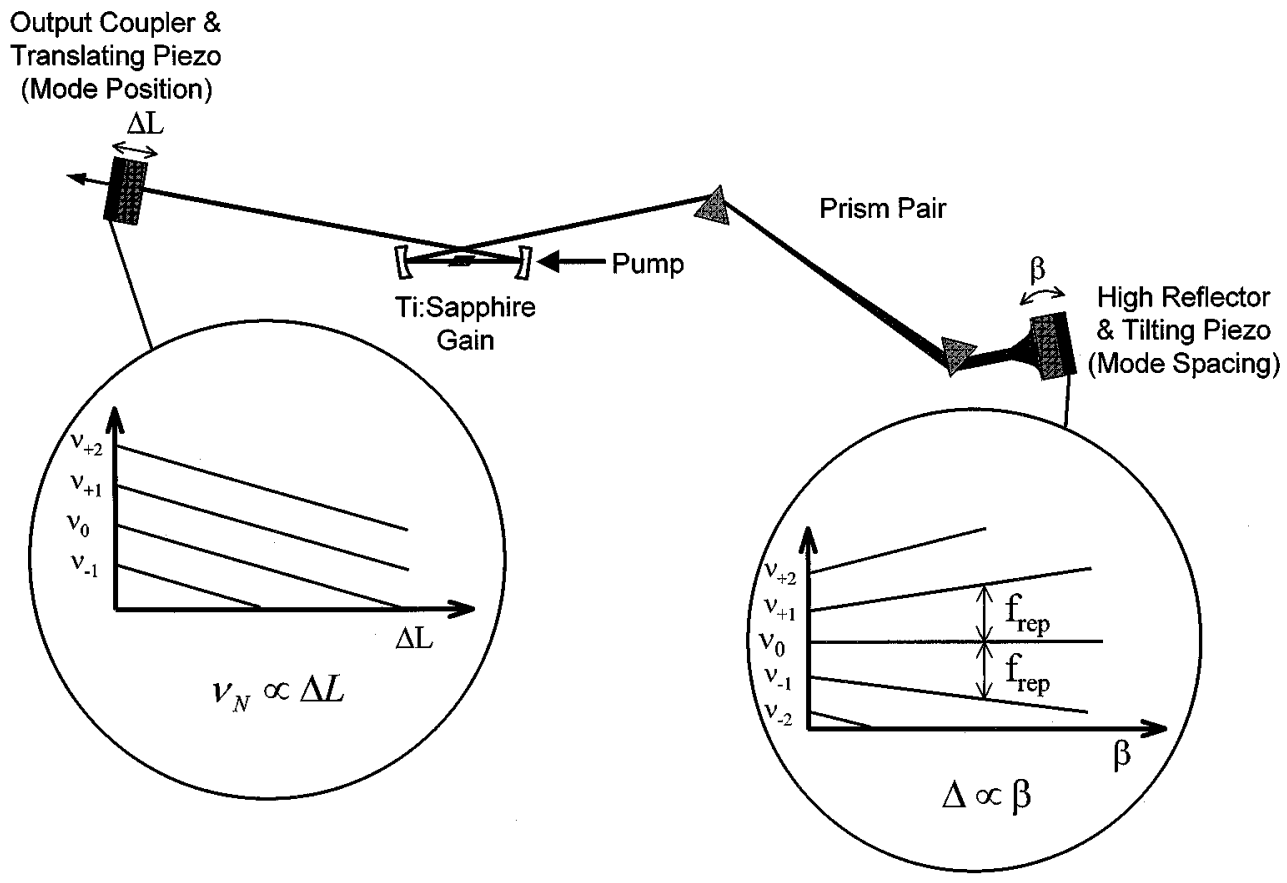

Fig. 3. Femtosecond laser stabilization. Modes are shifted the same amount fractionally by translating PZT and the same amount differentially by twisting mirror.

\section{B. Broadening the Optical Bandwith With NonLinear Photonic Crystal Fiber}

But a serious bandwidth increase came by using the microstructure fiber [39] generously provided by J. Ranka and R. Windeler at Lucent Bell Labs; the JILA group was immediately able to repeat their Bell Labs demonstration of octave bandwidth generation [40]. Using the highly stable Nd: YAG laser described before, we could measure the beat frequencies between the fundamental $1064 \mathrm{~nm}$ and a nearby comb-line and, at the same time, with a second detector, the beat between that CW laser's second harmonic and a nearby comb line in the green. Knowing the approximate 532-nm frequency already from previous measurements allows one to find the integer comb order numbers, with confirmation from the signs of the beats and/or changing the pulse repetition frequency. Basically this measurement gives us the CW Nd: YAG laser frequency $\omega$, as the difference between the fundamental $\omega$ and the second harmonic $2 \omega$, which interval is then measured in terms of the comb-line spacing. This spacing is rigorously the femtosecond laser's pulse repeat rate, which we were controlling via a stable $\mathrm{Rb}$ clock guided relative to NIST UTC via common-view GPS. (Now a commercial Cs clock provides even better stability, with the same accuracy confirmation technique.) In essence this was like the Garching 1999 frequency metrology scheme, but this time only the stable reference laser and a single broadband femtosecond laser was required. We measured the $\mathrm{HeNe} 633-\mathrm{nm}$ laser and the Rb-stabilized laser at $778 \mathrm{~nm}$, in addition to the iodine-stabilized doubled Nd: YAG [48].

One clear family of concerns for frequency metrology involves the characteristics of the femtosecond laser itself: the pulse rep-rate stability and the optical frequency stability. It is interesting and potentially important that the former is actually difficult to measure completely: that is, at short times, the laser repetition rate is more stable than the best sources available in the RF domain. Thus, after frequency multiplication into the visible domain, customary RF sources are not useful as a means to stabilize the laser in the short term. But a slow servo control of the repetition rate is quite helpful in reducing the laser frequency noise and drift resulting from laboratory vibrations and thermal drift of the laser cavity for Fourier frequencies below $\sim 300 \mathrm{~Hz}$ where the equivalent levels exceed those of the RF reference. In an optically referenced approach, it is natural to establish tight locks of comb lines in the IR and green to the nearby 1064- and 532-nm stable frequencies from our iodine-stabilized Nd: YAG laser (see Fig. 4). For this locking, each of the heterodyne beats is regenerated with a phase-locked tracking voltage-controlled oscillator, which could be shortened to phase-tracking oscillator (PTO). Its output is a constant amplitude sinewave with its phase dynamically tracking the apparent phase of the beat signal plus its noise. Ordinarily, we find the SNR is $>20 \mathrm{~dB}$, i.e., some $>10 \mathrm{~dB}$ above the 10.6-dB threshold of signal/noise within the PTO's servo bandwidth (typically $30 \mathrm{kHz}$ ) which represents the locking threshold for $<1$ cycle slip/s [49]. At present, we can lock the 1064-nm beat within $<1 \mathrm{~Hz}$ using the "length" PZT, while the 532-nm beat frequency noise can simultaneously be held to $\sim 180 \mathrm{~Hz}$ or less using the "twister" PZT. This results in some 4 million stable lasers covering an octave bandwidth, with the least stable of these being $\sim 180 \mathrm{~Hz}$ for the ones near the 532-nm end [50]. New control-mode orthogonalization concepts are expected to improve this situation.

\section{Self-Referencing Femtosecond Laser}

An important possibility brought by the octave bandwidth is the capability to directly measure and stabilize the comb's offset frequency $f_{\text {ceo }}$. In this approach, with a BBO crystal we obtain the doubled frequencies of a number of modes from the 


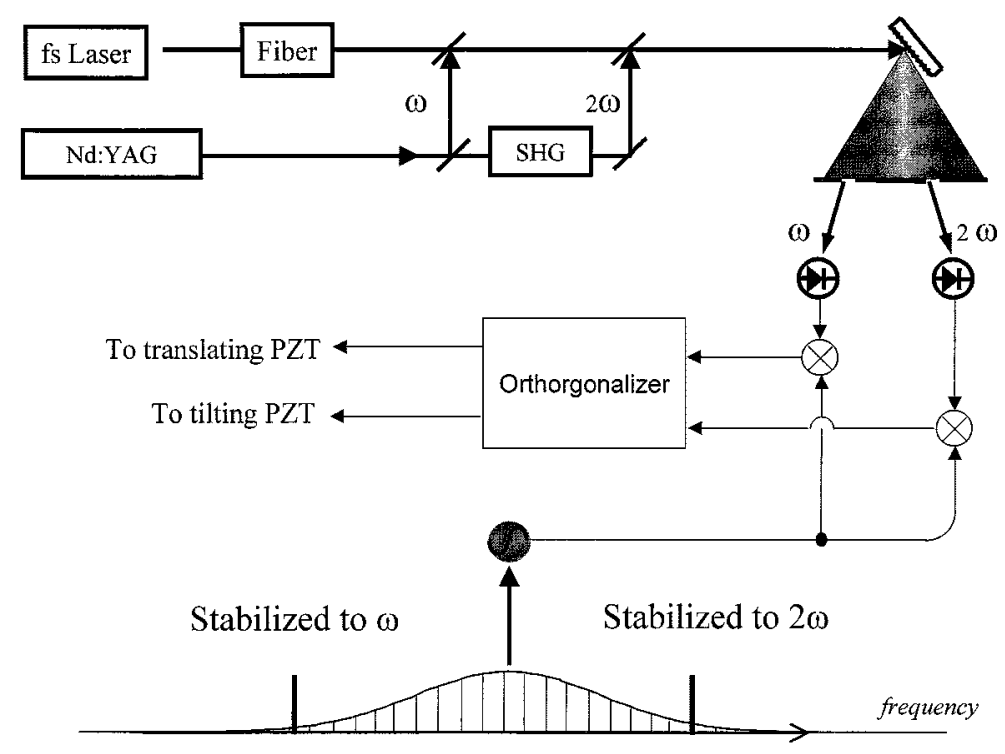

Fig. 4. Locking the entire femtosecond comb by using a super-stable optical frequency reference.

IR end of the spectrum. After doubling, these comb frequencies can be heterodyned with comb lines near the green limit of the PCF's output. In this photodetector's output, we find the difference of optical frequencies: $2 *\left(N f_{\text {rep }}+f_{\text {ceo }}\right)-\left(2 N f_{\text {rep }}+\right.$ $\left.f_{\text {ceo }}\right)=f_{\text {ceo. }}$. A nice scheme is to stabilize this offset frequency to be a rational fraction of the repetition rate [51]. From the frequency standard's viewpoint, basically any stable frequency could be chosen. However, from the time-domain viewpoint, the choice of $f_{\text {ceo }}$ as a phase-coherent submultiple of $f_{\text {rep }}$ is interesting because it leads to a periodicity of the pulse's carrier-envelope offset phase, with the pulse waveform repeating exactly after $m=f_{\text {rep }} / f_{\text {ceo }}$ pulses. Now the optical output pulse stream does have a strict periodicity and so can be represented by a Fourier harmonic series with a repetition rate a factor $m$ below the basic pulse rate. In other words, the frequency offset of the comb system is now controlled to be $f_{\text {ceo }}=$ $f_{\text {rep }} / m$. Fig. 5 shows the schematic of this measurement and stabilization system [51]. Note the use of an acousto-optic modulator in one arm to frequency shift the spectrum and thereby avoid the excess noise at dc. With digital frequency-based control of the phase slip per femtosecond pulse, we could confirm the stabilization by measuring the corresponding time domain "carrier-envelope phase" change per pulse. By cross-correlating one pulse with its second neighbor, the expected phase-step increments were clearly demonstrated [51]. Thus, we have the tools for some physics experiment which may display some sensitivity to the "carrier-envelope phase," in that it can be supplied with selected pulses with any choice of 16 phase-steps set across 2 pi. Speaking on the frequency domain side again, it is worth emphasizing again that this system represents a "gear-tight" optical-to-microwave phase-locked connection, ready to synthesize millions of optical frequencies from a suitably stable RF input. A suitable description of this RF/optical clockworks system may be to say it is "Self-Calibrating." Basically, for the $m=0$ setting, all optical output comb frequencies will be exact harmonics of the input RF frequency.

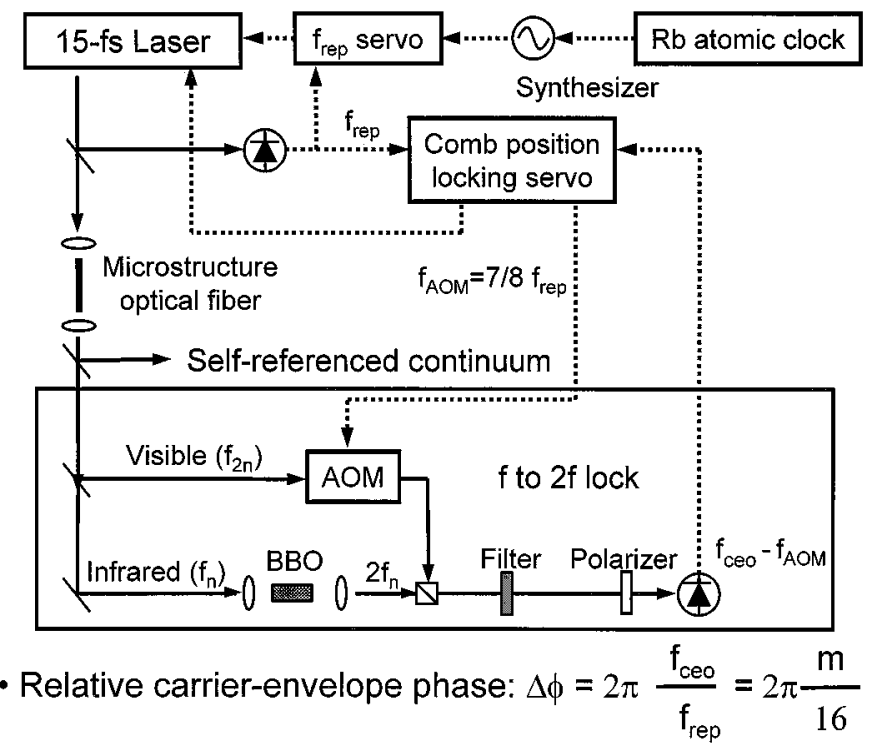

Fig. 5. Self-referencing optical frequency synthesizer. By heterodyne measurement of a cluster of green comb lines present in fiber-broadened output spectrum against green comb lines generated by frequency doubling the fiber's IR output, we recover the femtosecond laser comb's frequency offset from true harmonic comb position. Servos control the laser offset and repetition rate to produce an effective RF-to-visible harmonic generator.

\section{Summary of JILA Measurements Using Combs}

One useful effort has been the measurement at JILA of many of the "traditional" optical frequency references. So far, this includes the $\mathrm{HeNe} / \mathrm{I}_{2}$ stabilized laser at $633 \mathrm{~nm}$, two Rb-stabilized lasers at $778 \mathrm{~nm}$, and the $1064 \mathrm{~nm} / 532 \mathrm{~nm} \mathrm{Nd}$ : YAG laser stabilized via strong resonances in $\mathrm{I}_{2}$. All of these transitions have been measured previously, typically by means of "secondary" frequency standards such as the $\mathrm{CO}_{2}-\mathrm{OsO}_{4}$ system at $10.6 \mu \mathrm{m}$. In Fig. 6, we summarize these JILA frequency measurements made using "femtosecond comb technology." In all cases, one finds agreement with the values promulgated by the accepted 


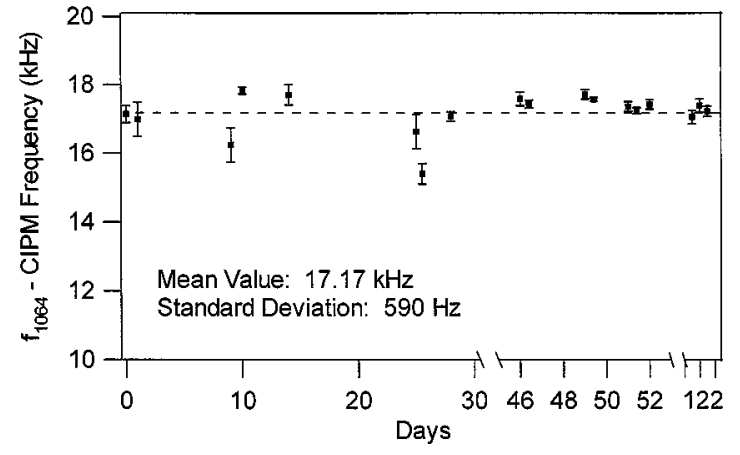

(a)

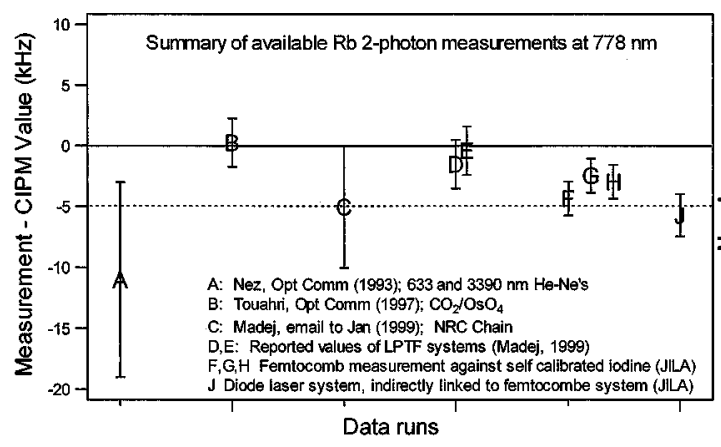

(c)

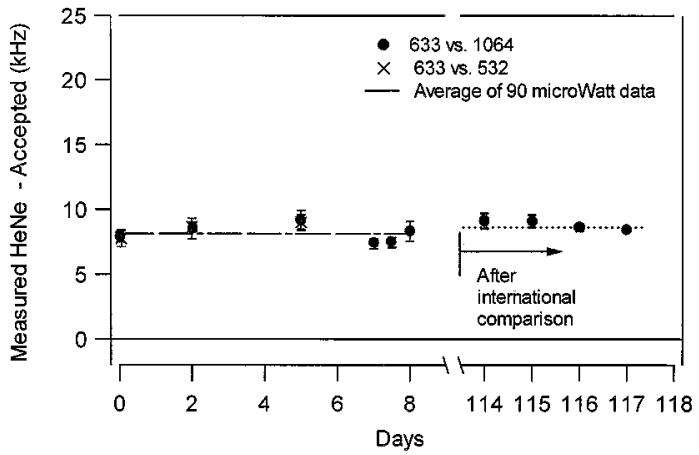

(b)

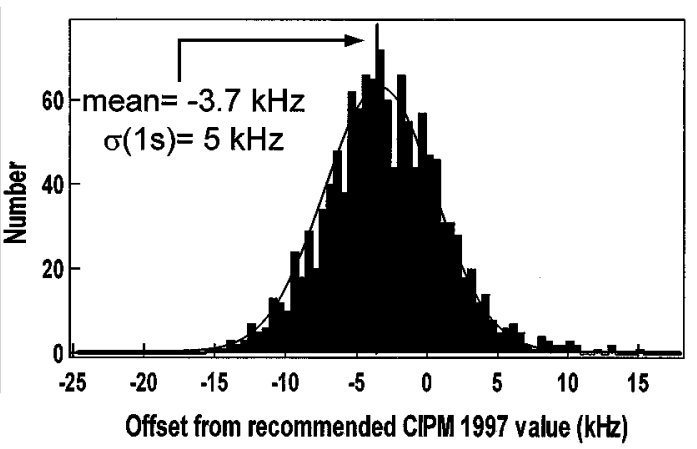

(d)

Fig. 6. Frequency measurements made with the femtosecond laser comb. All offsets referred to CIPM recommended values. (a) Frequency offset of green 532-nm I $\mathrm{I}_{2}$ component $a_{10}$ in R(56) 32-0 band. (b) Measured frequency of $\mathrm{I}_{2}$-stabilized 633-nm HeNe laser. (c) Frequency of 778-nm diode laser stabilized via Rb two-photon absorption. (d) Histogram of Rb measurements. Almost all the width arises from FM noise of Rb-based RF reference clock.

"experts" in the field, those scientists charged with this optical frequency metrology in the national metrology labs of basically all of the developed countries. Collectively, this Consultative Committee for Length (CCL) serves under the aegis of the International Bureau of Weights and Measures, located in Sèvres, France, just outside of Paris. Their most recent document [52] provides recommended values - and uncertainties - of frequencies for a large number of stabilized reference lasers. This document can be expected to be importantly upgraded following this September's meeting when all the new femtosecond combbased measurements will be considered.

Of course, the major labs can dedicate more effort to the maintenance and improvement of these working standards, with the result that, by employing extra care and performing auxiliary measurements, the CCL-published uncertainties can often be effectively reduced. Such working uncertainties may be threefold to fivefold smaller than the CCL recommended ones. Interestingly, one finds uniformly by our measurements that the CCLstated uncertainties values are sufficient. The CCL (2001) members will note that the shifts of frequency required in their future recommendations document (analogous to [52]) are just the same order as the "reduced" uncertainty these specialists might regard to be made possible by extra careful work. The HeNe value, resulting from a collaboration with the NRC (Ottawa) and the International Bureau of Weights and Measures (Paris), was reported recently [53]. It was possible to show equivalence of the JILA femtosecond comb and the NRC chain results at the sub-kilohertz level, basically using a well-maintained $\mathrm{HeNe} / \mathrm{I}_{2}$ laser as a transfer standard. Connection via the International
Bureau of Weights and Measures transfer lasers shows that the nominal frequency of the "as maintained" HeNe reference needs to be revised upward by $\sim 7 \mathrm{kHz}$.

\section{E. The Possibilities of Frequency Errors}

A fundamental issue for the use of such a comb-based "clockwork" mechanism for physical measurement is the question whether there could be, in some unexpected way, a possibility that the predicted frequency relationships are not quite exact, meaning that there would be some kind of frequency errors in the comb output. The Garching group has presented a number of tests based on different physical comparisons, such as a femtosecond laser comb versus a Kourogi comb, and several tests using interval divider stages [43]. Of course, no problem has been discovered: all measurements show the accuracy to be ideal to within the measurement precision, which in some cases has been reported to be in the $10^{-16}$ range or even less. Another profitable avenue for testing the comb accuracy issue can be based on physical argument. For example, surely we do not expect to find a different mode spacing/repetition rate coming from the fiber output end as a function of wavelength, for example, of the emitted light. Some number of pulses enter the fiber each second and, if there is no damage or other accumulative process underway in the fiber, surely we can expect the same number of emitted pulses each second. However, possibly the broad comb formed in these nonlinear interactions could not be representable as a single comb: we consider that the frequency offset $f_{\text {ceo }}$ could be different as a function of wavelength. Two scenarios suggest themselves immediately. If the process causing the color 
conversion were not step-by-step, but were rather discontinuous across some wavelength gap, perhaps the carrier-envelope offset frequency of the second partial comb could have another value. For example, at high excitations one can observe that blue light is present in the fiber within a few millimeters, while the useful light we need at $532 \mathrm{~nm}$ seems to develop more slowly. Or Raman scattering to the red, followed by cross-phase modulation, could also generate an isolated comb portion with a shifted offset $f_{\mathrm{ceo}}$. However, in exploring the obtained frequency values while the laser intensity is changed, one finds no detectable effect of this type. Another concern could be that the fiber has been seriously shocked by the passage of the powerful main pulse: atoms are still dephasing from the coherent superposition states formed to physically accomplish the intensity-dependent phaseshift. Probably the green light is always slightly late leaving near the output end of the fiber, trailing the higher velocity 800 -nm primary pulse. Always each green pulse will find the fiber in the process of relaxing back to a thermal situation. If the index of refraction were then to be ramping back down toward the calm fiber limit, perhaps we can expect the green portion of the spectrum to have a blue-shifted value of $f_{\text {ceo }}$, relative to the main spectrum. A test of this idea using an interval divider scheme and intermediate 709-nm laser is being prepared. The equivalent Garching test showed no problems, but the utilized frequency range was less and the length of material experiencing the powerful pulse was much reduced ( $2 \mathrm{~mm}$ of Ti: sapphire crystal versus $15 \mathrm{~cm}$ of microstructure silica fiber). At this point, the summary of all experience to date is that no accuracy problem whatsoever has been detected for frequency measurements.

In practice, starting with a microwave standard to obtain a known optical comb output, we noted already that it will be useful to make this repetition rate locking with a compromise speed, in order to conserve the superior short-term stability of the laser. Indeed, in the future, it will likely be the reverse direction which will be of interest for metrology, as the short and medium-term performance of optical standards based on ultranarrow optical resonances already match or exceed nearly all RF sources [31]. Of course, the present standard-the Cs atomic beam standard since 1967 - is the Cs microwave transition at 919, 263, and $1770 \mathrm{~Hz}$. While atomic fountain techniques have lengthened the interaction time to $\sim 1 \mathrm{~s}$, new accuracy-degrading problems appear likely to provide an accuracy limit $\sim 1.5 \times 10^{-15}$ [54]. How long will it be until all this optical resonance and laser technology will bring us a new definition of the second? The near future will be rich indeed.

\section{Closing}

Regarding the existence of a true "Optical Clock," we are not quite ready yet to deliver the "true line-center" information that will make this second paradigm shift possible or mandatory. For fundamental standards, one good avenue is clear: we simply learn to deal with resonances of increasingly narrow widths. (For example, we have recently learned from NIST colleagues that frequency repeatability of a few $\times 10^{-15}$ has been obtained over months with the $\mathrm{Hg}^{+}$resonance.) This narrow-line approach was noted earlier, with $\mathrm{Yb}^{+}$and its 10-year lifetime representing the penultimate limit. Still, one wonders if it would be possible to learn to deal with the appreciably broader resonances we can obtain in a simple cell; as shown in Fig. 2 already one can obtain impressive stability, reaching into the mid $10^{-15}$ domain. But the long-term stability and frequency repeatability are just not yet at the levels needed: rarely do we find an optical system in which the stability is still nicely improving with averaging time beyond, say, $1000 \mathrm{~s}$. An equivalent statement is that we can make a complete readjustment of the system optics and electronics and will find a new lock frequency, offset typically by an amount which corresponds to the stability value at a few seconds' integration time, or less. Even more instructive is to have an intelligent and skilled colleague in another lab build his "dream" system and bring it to our lab for an "intercomparison" to test the independent reproducibility of systems based on the "best practice" of two independent labs [55]. Invariably, the frequency offset between the two systems is larger than one could have expected-and not by just $\sqrt{2}$.

What are the main reasons behind this experience? Realistically, the art of stabilized lasers is not yet complete. Sometimes the differences arise from engineering deficiencies, while from time to time still another physics "gottcha" shows up. In design, one direction leads toward high SNR by using a strong line, higher gas pressure, and higher laser power. Here, one can find a broader resonance, more robust operation, and a smooth but large dependence of frequency shift upon operating parameters. Defects of modulation purity and modulation width-dependent shifts (associated with a small asymmetry of the resonance profile) are the usual line center reproducibility limits. The classic case here is the ubiquitous HeNe red laser stabilized with molecular iodine. Except that the usual line is not at all strong, but it is broad ( $\sim 5 \mathrm{MHz})$ and, of course, convenient. A stability of $1 \times 10^{-11}$ is obtained at $1 \mathrm{~s}$ and the best performance approaches $1 \times 10^{-13}$ at $10^{4} \mathrm{~s}$. Reproducibility is $\sim 5 \times 10^{-12}$ [53]. The next performance step may be the iodine-stabilized $\mathrm{Nd}$ : YAG frequency-doubled laser. Now the operating linewidth is 0.5 rather than $5 \mathrm{MHz}$. Because it is a very strong absorbing line, the pressure may be reduced more than an order of magnitude. This leads to a stability of $\sim 5 \times 10^{-14}$ at $1 \mathrm{~s}$, improving to $4 \times 10^{-15}$ at $500 \mathrm{~s}$ [27]. The lower linewidth means that any modulation problems are smaller because the modulation width is reduced, and the remaining errors influence a narrower line. A frequency reproducibility of $1 \times 10^{-12}$ is straightforward and can readily be made twofold or threefold better.

Looking at the future, it will be interesting to try tunable lasers with the longer lived iodine transitions to higher vibrational levels, as the upper level lifetimes are known to increase dramatically as the dissociation limit is approached. Toward this end, we have already built such a system and discovered in iodine some interesting sub-Doppler spectra near $500 \mathrm{~nm}$ [56]. The molecular overtones with expanded beams will be interesting. And a few "forbidden" lines beckon us ....

\section{ACKNOWLEDGMENT}

The authors are pleased to acknowledge their interactions with many colleagues in this work. Particularly precious is the goodwill and cooperation between groups that has made this new epoch possible. Long-term NIST colleagues L. Hollberg 
and C. Oates and their colleagues generously shared their ideas and remarkable Ca results with the authors, as did J. Bergquist and his group with their spectacular $\mathrm{Hg}^{+}$results. $\mathrm{K}$. Vogel and S. Diddams participated in some of the recent NIST work and were, along with D. Jones, enthusiastic and valuable contributors in earlier phases of the authors' JILA work. Other indispensable JILA colleagues are H. Kapteyn and M. Murnane, who have patiently helped us come up to speed with the femtosecond sources and background knowledge. The JILA authors enthusiastically thank their colleagues at the Max Planck Institute for Quantum Optics (Garching) for their generous and open exchange of ideas, information, and people. Collaboration across the Atlantic with Prof. Hänsch's group has been materially aided by an active exchange quantum (scientist) known as T. Udem: they thank him, his boss, and their colleagues for a great run. Of course, the authors would be nowhere without the broad spectrum enabled by the generous contribution of some "Rainbow Fiber" by J. Ranka, R. Windeler, and A. Stentz of Lucent Technology/Bell Labs. There surely are many other people who have contributed important ideas, talents, and results either directly or into the worldwide knowledge base. If, regrettably, this brief paper has omitted proper reference to their work, the authors hope they can take comfort and even celebrate what they all collectively have achieved in the last several years: optical frequency measurement has come of age!

\section{REFERENCES}

[1] J. L. Hall, "The laser absolute wavelength standard problem," IEEE J. Quantum Electron., vol. QE-4, pp. 638-641, 1968.

[2] F. Schmidtkaler, D. Leibfried, S. Seel, C. Zimmermann, W. Konig, M. Weitz, and T. W. Hansch, "High-resolution spectroscopy of the 1s-2s Transition of Atomic-Hydrogen and Deuterium," Phys. Rev. A, vol. 51, pp. 2789-2800, 1995

[3] D. L. Shiner and R. Dixson, "Measuring the fine-structure constant using Helium fine-structure," IEEE Trans. Instrum. Meas., vol. 44, pp. 518-521, 1995

[4] D. J. Berkeland, J. D. Miller, J. C. Bergquist, W. M. Itano, and D. J. Wineland, "Laser-cooled mercury ion frequency standard," Phys. Rev. Lett., vol. 80, pp. 2089-2092, 1998.

[5] R. J. Rafac, B. C. Young, J. A. Beall, W. M. Itano, D. J. Wineland, and J. C. Bergquist, "Sub-dekahertz ultraviolet spectroscopy of $\mathrm{Hg}-199(+), "$ Phys. Rev. Lett., vol. 85, pp. 2462-2465, 2000.

[6] J. von Zanthier, J. Abel, T. Becker, M. Fries, E. Peik, H. Walther, R. Holzwarth, J. Reichert, T. Udem, T. W. Hansch, A. Y. Nevsky, M. N. Skvortsov, and S. N. Bagayev, "Absolute frequency measurement of the In-115(+) 5s(2 1)S(0)-5s5p P-3(0) transition," Opt. Commun., vol. 166, pp. 57-63, 1999.

[7] J. E. Bernard, A. A. Madej, L. Marmet, B. G. Whitford, K. J. Siemsen, and S. Cundy, "Cs-based frequency measurement of a single, trapped ton transition in the visible region of the spectrum," Phys. Rev. Lett., vol. 82, pp. 3228-3231, 1999.

[8] M. Roberts, P. Taylor, G. P. Barwood, W. R. C. Rowley, and P. Gill, "Observation of the S-2(1/2)-F-2(7/2) electric octupole transition in a single Yb-171(+) ion," Phys. Rev. A, vol. 6202, no. 020501, p. 0501, 2000.

[9] O. Poulsen and J. L. Hall, "Spectroscopic investigations in Bi-209-I using tunable-Cw-dye-laser spectroscopy," Phys. Rev. A, vol. 18, pp. 1089-1096, 1978.

[10] G. Uhlenberg, J. Dirscherl, and H. Walther, "Magneto-optical trapping of silver atoms," Phys. Rev. A, vol. 6206, no. 063404, p. 3404, 2000.

[11] H. Schnatz, B. Lipphardt, J. Helmcke, F. Riehle, and G. Zinner, "First phase-coherent frequency measurement of visible radiation," Phys. Rev. Lett., vol. 76, pp. 18-21, 1996.

[12] C. W. Oates, E. A. Curtis, and L. Hollberg, "Improved short-term stability of optical frequency standards: Approaching $1 \mathrm{~Hz}$ in $1 \mathrm{~s}$ with the Ca standard at 657 nm," Opt. Lett., vol. 25, pp. 1603-1605, 2000.
[13] F. Ruschewitz, J. L. Peng, H. Hinderthur, N. Schaffrath, K. Sengstock, and W. Ertmer, "Sub-kilohertz optical spectroscopy with a time domain atom interferometer," Phys. Rev. Lett., vol. 80, pp. 3173-3176, 1998.

[14] H. Katori, T. Ido, Y. Isoya, and M. Kuwata-Gonokami, "Magneto-optical trapping and cooling of strontium atoms down to the photon recoil temperature," Phys. Rev. Lett., vol. 82, pp. 1116-1119, 1999.

[15] K. R. Vogel, T. P. Dinneen, A. Gallagher, and J. L. Hall, "Narrow-line Doppler cooling of strontium to the recoil limit," IEEE Trans. Instrum. Meas., vol. 48, pp. 618-621, 1999.

[16] M. Roberts, P. Taylor, G. P. Barwood, P. Gill, H. A. Klein, and W. R. C. Rowley, "Observation of an electric octupole transition in a single ion," Phys. Rev. Lett., vol. 78, pp. 1876-1879, 1997.

[17] M. Niering, R. Holzwarth, J. Reichert, P. Pokasov, T. Udem, M. Weitz, T. W. Hansch, P. Lemonde, G. Santarelli, M. Abgrall, P. Laurent, C. Salomon, and A. Clairon, "Measurement of the hydrogen 1S-2S transition frequency by phase coherent comparison with a microwave cesium fountain clock," Phys. Rev. Lett., vol. 84, pp. 5496-5499, 2000.

[18] T. C. Killian, D. G. Fried, L. Willmann, D. Landhuis, S. C. Moss, T. J. Greytak, and D. Kleppner, "Cold collision frequency shift of the 1S-2S transition in hydrogen," Phys. Rev. Lett., vol. 81, pp. 3807-3810, 1998.

[19] A. Huber, T. Udem, B. Gross, J. Reichert, M. Kourogi, K. Pachucki, M. Weitz, and T. W. Hansch, "Hydrogen-deuterium 1S-2S isotope shift and the structure of the deuteron," Phys. Rev. Lett., vol. 80, pp. 468-471, 1998.

[20] M. K. d. L. Nakagawa, Y. Awaji, and M. Kourogi, "Accurate optical frequency atlas of the $1.5-\mu \mathrm{m}$ bands of acetylene," J. Opt. Soc. Amer. B, vol. 13, pp. 2708-2714, 1996.

[21] J. L. Hall, C. J. Borde, and K. Uehara, "Direct optical resolution of recoil effect using saturated absorption spectroscopy," Phys. Rev. Lett., vol. 37, pp. 1339-1342, 1976

[22] C. J. Borde, J. L. Hall, C. V. Kunasz, and D. G. Hummer, "Saturated absorption-line shape-calculation of transit-time broadening by a perturbation approach," Phys. Rev. Lett., vol. 14, pp. 236-263, 1976.

[23] L. S. Ma, J. Ye, P. Dube, and J. L. Hall, "Ultrasensitive frequency-modulation spectroscopy enhanced by a high-finesse optical cavity: Theory and application to overtone transitions of $\mathrm{C}_{2} \mathrm{H}_{2}$ and $\mathrm{C}_{2} \mathrm{HD}$," J. Opt. Soc. Amer. B, vol. 16, pp. 2255-2268, 1999.

[24] S. N. Bagayev, A. E. Baklanov, V. P. Chebotayev, and A. S. Dychkov, "Superhigh resolution spectroscopy in methane with cold molecules," Appl. Phys. B, vol. 48, pp. 31-35, 1989.

[25] J. Ye, L. S. Ma, and J. L. Hall, "Ultrasensitive detections in atomic and molecular physics: Demonstration in molecular overtone spectroscopy," J. Opt. Soc. Amer. B, vol. 15, pp. 6-15, 1998.

[26] - "High-resolution frequency standard at $1030 \mathrm{~nm}$ for $\mathrm{Yb}$ : YAG solid-state lasers," J. Opt. Soc. Amer. B, vol. 17, pp. 927-931, 2000.

[27] J. Ye, L. Robertsson, S. Picard, L. S. Ma, and J. L. Hall, "Absolute frequency atlas of molecular $\mathrm{I}_{2}$ lines at $532 \mathrm{~nm}$, , IEEE Trans. Instrum. Meas., vol. 48, pp. 544-549, 1999.

[28] J. L. Hall, L. S. Ma, M. Taubman, B. Tiemann, F. L. Hong, O. Pfister, and J. Ye, "Stabilization and frequency measurement of the $\mathrm{I}_{2}$-stabilized $\mathrm{Nd}$ : YAG laser," IEEE Trans. Instrum. Meas., vol. 48, pp. 583-586, 1999.

[29] F.-L. Hong, J. Ye, L.-S. Ma, S. Picard, C. J. Bordé, and J. L. Hall, "Rotation dependence of electric quadrupole hyperfine interaction in the ground state of molecular iodine by high-resolution laser spectroscopy," J. Opt. Soc. Amer. B, vol. B 18, p. 379, 2001.

[30] C. Daussy, F. Ducos, G. D. Rovera, and O. Acef, "Performances of $\mathrm{OsO}_{4}$ stabilized $\mathrm{CO}_{2}$ lasers as optical frequency standards near $29 \mathrm{THz}$," IEEE Trans. Ultrason., Ferroelect., Freq. Contr., vol. 47, pp. 518-521, 2000.

[31] Th. Udem, S. A. Diddams, K. R. Vogel, C. W. Oates, E. A. Curtis, W. D. Lee, W. M. Itano, R. E. Drullinger, J. C. Bergquist, and L. Hollberg, "Absolute frequency measurements of the $\mathrm{Hg}^{+}$and $\mathrm{Ca}$ optical clock transitions with a femtosecond laser," Phys. Rev. Lett., vol. 86, pp. 4996-4999, 2001.

[32] D. A. Jennings, K. M. Evenson, and D. J. E. Knight, "Optical frequency measurements," Proc. IEEE, vol. 74, pp. 168-179, 1986.

[33] D. J. E. Knight, "Present frequency standards for lasers," in European Frequency and Time Forum, Neuchatel, Switzerland, 1997.

[34] T. Udem, A. Huber, M. Weitz, D. Leibfried, W. Konig, M. Prevedelli, A. Dimitriev, H. Geiger, and T. W. Hansch, "Phase-coherent measurement of thehydrogen $1 \mathrm{~S}-2 \mathrm{~S}$ frequency with an optical frequency interval divider chain," IEEE Trans. Instrum. Meas., vol. 46, pp. 166-168, 1997.

[35] H. R. Telle, D. Meschede, and T. W. Hansch, "Realization of a new concept for visible frequency-division-phase locking of harmonic and sum frequencies," Opt. Lett., vol. 15, pp. 532-534, 1990.

[36] D. E. Spence, P. N. Kean, and W. Sibbett, "60-Fsec pulse generation from a self-mode-locked Ti-sapphire laser," Opt. Lett., vol. 16, pp. 42-44, 1991. 
[37] F. F., M. E. Krausz, T. Brabec, P. F. Curley, M. Hofer, M. H. Ober, C. Spielmann, E. Wintner, and A. J. Schmidt, "Femtosecond solid-state lasers," IEEE J. Quantum Electron., vol. 28, pp. 2097-2122, 1992.

[38] M. T. Asaki, C. P. Huang, D. Garvey, J. P. Zhou, H. C. Kapteyn, and M. M. Murnane, "Generation of 11-fs pulses from a self-mode-locked Ti-sapphire laser," Opt. Lett., vol. 18, pp. 977-979, 1993.

[39] J. K. Ranka, R. S. Windeler, and A. J. Stentz, "Optical properties of high-delta air-silica microstructure optical fibers," Opt. Lett., vol. 25, pp. 796-798, 2000

[40] - "Visible continuum generation in air-silica microstructure optical fibers with anomalous dispersion at $800 \mathrm{~nm}$," Opt. Lett., vol. 25, pp. $25-27,2000$

[41] J. N. Eckstein, A. I. Ferguson, and T. W. Hansch, "High-resolution 2-photon dpectroscopy with picosecond light-pulses," Phys. Rev. Lett., vol. 40, pp. 847-850, 1978.

[42] T. Udem, J. Reichert, R. Holzwarth, and T. W. Hansch, "Absolute optical frequency measurement of the cesium $\mathrm{D}_{1}$ line with a mode-locked laser," Phys. Rev. Lett., vol. 82, pp. 3568-3571, 1999.

[43] J. Reichert, R. Holzwarth, T. Udem, and T. W. Hansch, "Measuring the frequency of light with mode-locked lasers," Opt. Commun., vol. 172, pp. 59-68, 1999.

[44] R. L. Fork, O. E. Martinez, and J. P. Gordon, "Negative dispersion using pairs of prisms," Opt. Lett., vol. 9, pp. 150-152, 1984.

[45] K. F. Kwong, D. Yankelevich, K. C. Chu, J. P. Heritage, and A. Dienes, "400-Hz mechanical scanning optical delay line," Opt. Lett., vol. 18, pp. 558-560, 1993

[46] S. A. Diddams, D. J. Jones, L. S. Ma, S. T. Cundiff, and J. L. Hall, "Optical frequency measurement across a 104-THz gap with a femtosecond laser frequency comb," Opt. Lett., vol. 25, pp. 186-188, 2000.

[47] D. J. Jones, S. A. Diddams, M. S. Taubman, S. T. Cundiff, L. S. Ma, and J. L. Hall, "Frequency comb generation using femtosecond pulses and cross-phase modulation in optical fiber at arbitrary center frequencies," Opt. Lett., vol. 25, pp. 308-310, 2000.

[48] S. A. Diddams, D. J. Jones, J. Ye, T. Cundiff, J. L. Hall, J. K. Ranka, R. S. Windeler, R. Holzwarth, T. Udem, and T. W. Hansch, "Direct link between microwave and optical frequencies with a $300 \mathrm{THz}$ femtosecond laser comb," Phys. Rev. Lett., vol. 84, pp. 5102-5105, 2000.

[49] J. L. Hall, M. S. Taubman, S. A. Diddams, B. Tiemann, J. Ye, L.-S. Ma, D. Jones, and S. T. Cundiff, "Stabilizing and measuring optical frequencies," in Laser Spectroscopy (XIV Int. Conf.), vol. 1999, R. Blatt et al., Eds., 1999, pp. 51-60.

[50] J. Ye, J. L. Hall, and S. A. Diddams, "Precision phase control of an ultrawide-bandwidth femtosecond laser: A network of ultrastable frequency marks across the visible spectrum," Opt. Lett., vol. 25, pp. 1675-1677, 2000.

[51] D. J. Jones, S. A. Diddams, J. K. Ranka, A. Stentz, R. S. Windeler, J. L. Hall, and S. T. Cundiff, "Carrier-envelope phase control of femtosecond mode-locked lasers and direct optical frequency synthesis," Science, vol. 288, pp. 635-639, 2000.

[52] T. J. Quinn, "Practical realization of the meter (1997)," Metrologia, vol. 36, pp. 211-244, 1999

[53] T. H. Yoon, J. Ye, J. L. Hall, and J. M. Chartier, "Absolute frequency measurement of the iodine-stabilized $\mathrm{He}-\mathrm{Ne}$ laser at $633 \mathrm{~nm}$," Appl. Phys. B-Lasers and Optics, vol. 72, pp. 221-226, 2001.

[54] D. B. Sullivan, J. C. Bergquist, J. J. Bollinger, R. E. Drullinger, W. M. Itano, S. R. Jefferts, W. D. Lee, D. Meekhof, T. E. Parker, F. L. Walls, and D. J. Wineland, "Primary atomic frequency standards at NIST," $J$. Res. Nat. Inst. Stand. Technol., vol. 106, pp. 47-63, 2001.

[55] F. L. Hong, J. Ishikawa, J. Yoda, J. Ye, L. S. Ma, and J. L. Hall, "Frequency comparison of ${ }^{127} \mathrm{I}_{2}$-stabilized Nd : YAG lasers," IEEE Trans. Instrum. Meas., vol. 48, pp. 532-536, 1999.

[56] W.-Y. Cheng, T. H. Yoon, J. L. Hall, and J. Ye, , 2001.

[57] S. T. Cundiff, J. Ye, and J. L. Hall, "Optical frequency synthesis based on mode-locked lasers," Rev. Scientif. Instrum., vol. 72, pp. 3749-3771, 2001.

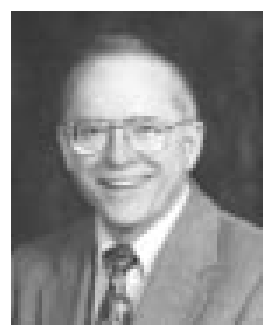

John L. Hall was born in 1934 in Denver, CO. He received the Ph.D. degree in physics from the Carnegie Institute of Technology, Pittsburgh, PA.

Since 1962, he has been with JILA (formerly the Joint Institute for Laboratory Astrophysics), Boulder, $\mathrm{CO}$, operated jointly by the University of Colorado, Boulder, and the National Institute of Standards and Technology (NIST). At JILA, he has been responsible for a number of major innovations and developments in laser frequency stabilization, high-resolution and ultrasensitive laser spectroscopy, laser cooling, quantum optics, and high-precision measurements using laser technology.

Dr. Hall is a Member of the National Academy of Sciences, a Senior Fellow of the NIST, and a Fellow of JILA, the Optical Society of America, and the American Physical Society.

Jun Ye was born in 1967 in Shanghai, China. He received the B.S. degree from Jiao Tong University, Shanghai, China, in 1989, the M.S. degree from University of Mexico at Albuquerque in 1991, and the Ph.D. degree from the University of Colorado, Boulder, in 1997.

He was an R. A. Millikan Prize Post-Doctoral Fellow, Quantum Optics Group, California Institute of Technology, Pasadena, until he joined JILA in 1999, where he recently became a Fellow. His research interests include high-precision measurements, high-resolution and ultrasensitive laser spectrosocopy, optical frequency metrology, femtosecond laser stabilization, cold molecules, and quantum dynamics in optical and atomic physics.

Scott A. Diddams was born in Gallup, NM, in 1967. He received the Ph.D. degree in optical science from the University of New Mexico at Albuquerque in 1996.

Between 1996 and 2000, he was a postdoctoral fellow at JILA, Boulder, CO, the National Institute of Standards and Technology (NIST), Boulder, CO, and the University of Colarado, Boulder. Currently, he is a Staff Physicist in the Time and Frequency Division of NIST. Much of his research relates to the measurement and control of optical fields for studies involving ultrafast phenomenon, nonlinear optics, and precision spectrosocopy and metrology.

Long-Sheng Ma was born in 1941 in Shanghai, China. He graduated from East China Normal University, Shanghai, in 1963, majoring in electronics and physics.

He studied laser propogation in atmosphere and laser monitoring of air polution until 1980. Since 1981, his research has been in the field of ultrasensitive and high-resolution laser spectroscopy, laser frequency stabilization, and molecular spectroscopy. He was a visiting Scholar at JILA (formerly the Joint Institute for Laboratory Astrophysics), Boulder, $\mathrm{CO}$, and the University of Colorado, Boulder, from 1981 to 1983, and a Guest Scientist at the Institute for Laser-Physics, Hamburg University, Hamburg, Germany, from 1992 to 1993. From 1993 to 1998, he was once again with JILA. His permanent position is as Professor, Department of Physics, East China Normal University, Shanghai.

Steven T. Cundiff, photograph and biography not available at the time of publication.

David J. Jones, photograph and biography not available at the time of publication. 